\title{
An Analysis of the Prospects for the Co-construction of Longjiang Drama and Art Colleges
}

\author{
Cheng Yimin \\ Heihe College, Heihe Heilongjiang 164300
}

Keywords: Longjiang opera; history; elements

Abstract: Longjiang Opera, a new type of drama born in 1959, has grown up for more than 40 years and become a spring blossom on the land of Guandong. To sum up its experience, it is necessary to consciously solve five elements: script, feature, innovation, talent and openness. These five elements are solved, and audience problems, market problems and brand problems can be solved easily.

\section{Introduction}

At the third meeting of the Northeast Cooperation Zone in 1958, Premier Zhou pointed out that "to prosper and develop the Northeast culture, we should create our own local opera." According to the instructions of the premier, the Longjiang opera was born in 1959 with the establishment of the Longjiang theatre. Now, the Longjiang opera has passed through the age of no doubt, and is now sitting in the pear garden in China with her mature beauty. After 7 years of experiment and 10 years of "Cultural Revolution", on the 25th anniversary of the founding of Longjiang Theater, A Theoretical Seminar on Longjiang Theater was held, and on the 30th anniversary of the founding of Longjiang Theater, a seminar on the construction and development of Longjiang Opera was held. In the latter important seminar, Heilongjiang Provincial Department of Culture, after full investigation and study, has issued a general view on the construction and development of Longjiang Opera. Under the guidance of this opinion, through the efforts of all the artists and managers of Longjiang Theater, especially the double struggle of Bai Shuxian in art and management, Longjiang Opera has made great progress. In June this year, the Chinese Drama Association, the Chinese Culture Daily, the Heilongjiang Provincial Party Committee's Yichuan Department and the Heilongjiang Provincial Department of Culture jointly held the "Bai Shuxian and Longjiang Drama Art Seminar". Nearly 20 experts and scholars from Beijing and more than 20 artistic theorists from our province gathered together to discuss the development of Longjiang Drama in the past 40 years. Deep discussion. We have reason to believe that Longjiang Opera, a new local drama, will develop healthily with the support of long-term artistic practice and scientific theory. The following are my thoughts on the construction and development of Longjiang opera.

\section{Many Kinds of Repertoire Experiments are Fertile Soil for Longjiang Opera to Mature}

To be recognized by the audience and by history, a local opera must have the following main 
conditions: first, a unique and distinctive musical aria; second, a group of talents with famous characters as the core of the opera; third, a large number of repertoire performances and their own conservative repertoire. Four, there is a set of theoretical system or experience crystallized for the generation and development of this drama. All mature local operas are equipped with the above main conditions. Among these conditions, "having a large number of performances" is the most important condition, because: the birth of a new local opera, like the birth of other new things, must have its birth of external causes; subjectively, must have its development of internal causes, which is the artist of the opera in the long-term. The artistic talent, artistic experience and artistic creativity that are generated and developed in art practice. The maturity and development of the new drama must follow the historical process of dialectical unity of practice and cognition. There is no new thing that suddenly matures in the morning, and there is no new drama that comes overnight. Because of this, many local operas have to go through a decade, a hundred years of grinding and maturity. Because of this, many local operas generally have more performance repertoire.

The so-called "having more repertoire" is only a synonym for the mature development of a drama in the long-term artistic practice and through the examination of the audience. It is precisely in the long-term practice of more performances, unique and distinctive Musical Singing will be formed; famous actors will stand out; a large number of artistic talent will appear; retained repertoire will be established; self-contained experience and theory will crystallize; and will form a more stable audience. The reason why Longjiang Opera came into being is not only because Premier Zhou paid close attention to the words of Northeast local operas, but also because she had the vigorous and mature mother body - Northeast local opera, Northeast folk music and folk dance. The reason why Longjiang Opera matures is not only because of her mother's innate abundance, but also because she has acquired a variety of internal reasons for the maturity of Longjiang Opera in many performances, that is, long-term artistic practice. Since its birth, Longjiang Opera has created and performed more than 80 plays in more than 40 years. This historical period and the number of repertoires can or can make a local opera mature. In the short seven years from the birth of Longjiang Opera to the eve of the Cultural Revolution, Longjiang Theatre has rehearsed more than 30 plays, through which it has made beneficial exploration in all aspects of script creation, music singing, performance director, stage art and comprehensive art, and has made gratifying achievements. Among them, the traditional drama "Five Girls", "Hanjiang Pass", "Shuangsuoshan", "Chunling Temple", and the modern drama "Li Shuangshuangzhi's" Mountains"and other repertoires have won the audience's welcome and praise. The exploration of these plays has laid a good foundation for the subsequent Longjiang opera experiment. After the Cultural Revolution, the Longjiang Theater has made a comprehensive summary of the experiment of Longjiang Opera in the 25 years since its establishment, and on this basis, launched a new round of experiment and construction of Longjiang Opera. In 1985, the theatre rehearsed the excellent traditional opera "heroine girl", its singing, playing and reciting are impressive. Then he rehearsed the modern drama "the absurd man of chaos" and "the waist of cotton trousers", These two plays have made great efforts in comprehensive art, especially in stage art. In 1985, he also rehearsed the play of "iron bow" and was well received. In the new round of Longjiang Opera experiment, Bai Shuxian, the outstanding actor of Longjiang Opera in his prime, inherited the experimental results and successful experience of Longjiang Opera, boldly avoided the detour of applying and relying on Beijing Opera in the experiment, and sought nutrition from the black earth culture and the mother body of Longjiang Opera with his outstanding artistic intuition. Finally, Bai Shuxian succeeded in "Shuangsuoshan". 


\subsection{The Play Created a Unique Black Earth Liu Jinding}

From the macro perspective, this play and this character can be regarded as a turning point in the Longjiang drama experiment. Because it has established or contained the aesthetic spirit or artistic style of Longjiang Opera, which is the beauty of rough. In 1988, the theatre rehearsed the traditional drama "Lanqiao" and the modern drama "Pass the Pass", which won many awards for their successful artistic exploration. In 1989, the theatre launched the "absurd treasure jade" in the experimental history of Longjiang Opera, which pushed the experiment of Longjiang Opera to a new stage of "taking oneself as the dominant factor and absorbing all the strong points". Since then, the legend of Mulan has been introduced again in 90s. These three plays constitute the "Longjiang drama Trilogy". The trilogy and its protagonist Bai Shuxian stand out, marking the Dragon Opera has finally come to fruition. Longjiang opera finally has its own repertoire and name. We look back on the historical fact that Longjiang Opera has matured in more than 80 repertoires rehearsed over the past 40 years, not to pile up historical facts for review, but to find out the general and special laws of the development of Longjiang Opera, so as to facilitate the continued development of Longjiang Opera.

\subsection{The Main Regularities About Longjiang Opera}

First, Longjiang Opera, like other operas, can only be mature and perfect in the long-term artistic practice and in the performance of a large number of experimental plays. The performance of the new play is the life of the theater, and the fertile soil for the play's maturity. Secondly, in the process of maturity of a local drama, the subjective conditions of self-shaping, self-struggle and self-transcendence of the famous characters are very important. A great variety of operas and a large number of performance practices can not naturally produce famous corners. On the basis of cultural nourishment and a lot of practice, a famous actor must give full play to the spirit of struggle and creativity, so that he can stand out in the best realm of subjective and objective unity, and because of his brilliant appearance and cohesive force, make the new drama become a popular cultural brand. Thirdly, the repertoire of local operas or high-quality repertoire can only be formed in more performances and long-term grinding. Quality is of course crucial, but quality is accumulated, there is not enough quantity there will be no higher quality, boutique is also a "longterm accumulation of results".

\section{Regional Characteristics and Root seeking Complex are the Vivid Veins of Longjiang Opera Style}

In the process of creating Longjiang Opera, whether it is based on Beijing Opera or any other culture, or on black earth art and local culture, is the most important aesthetic problem of MeiguanLongjiang Opera. If the problem is not solved well, other efforts in the construction of Longjiang opera are of no value. As early as in the seminar held on the 30th anniversary of the founding of the Longjiang Theater, in the concluding comments on the construction and development of Longjiang Opera issued by the Provincial Culture Department, the author has given such a position to Longjiang Opera on the noumenon of what kind of drama Longjiang Opera should be and the construction of Longjiang Opera. On the basis of folk dance, a new type of local opera has developed with strong local characteristics and adapts to the aesthetic requirements of contemporary audiences. And stressed that the construction of Longjiang opera can not leave the Northeast Local Opera and music, dance this matrix. While positioning Longjiang Opera in this way, it points out the general policy of the construction of Longjiang Opera, that is, "to take root in the foundation, to learn from all the strong points, to adapt to the times, and to be a family of its 
own." In my opinion, the orientation of Longjiang Opera and the construction policy of Longjiang Opera are correct. In the past 10 years, Longjiang Opera has carried out creative and pioneering experiments in accordance with this correct orientation and policy, and has achieved gratifying success. For the new development of Longjiang Opera in the new century, as well as for other sister art can learn from the experience, we need to make a brief demonstration of the positioning of Longjiang Opera from the aesthetic point of view, in order to purify the real value of Longjiang Opera in the experimental process.We hope you find the information in this template useful in the preparation of your submission.

\section{The Spirit of Innovation and the Opening of Art are the Pioneers of Longjiang Opera's Self Transcendence}

Since the new period, the development of Longjiang Opera has been pushed to a new stage with the artistic spirit of opening up rather than closing the door. Bai Shuxian starred in "Shuangsuoshan" another way, skillfully absorb the performance of the Northeast folk arts such as Errenzhuan, Yangko, so that Liu Jinding became a genuine Northeast girl, which can not but be said to be a bold innovation at that time. The large-scale Longjiang opera "Emperor's Relatives" not only has some relatively complete and innovative factors in the comprehensive art, but also has a certain depth of humanistic connotation, reflecting the playwright's extraordinary artistic courage. The play is also one of the more complete and literary Longjiang opera, the pace of innovation is greater, should be said to be "absurd Baoyu". It is not particularly surprising to say that Beijing opera and Vietnamese opera will become popular if they draw material from A Dream of Red Mansions. Because the elegance of Peking Opera and the gentleness and elegance of Vietnam Opera, as well as the artistic tradition of these two operas, are easier to match the theme and interest of A Dream of Red Mansions, or can be said to be original soup. But the Longjiang play is different. Longjiang opera is rough and Tsuchino. The style of Longjiang Opera may be close to the style of Water Margin and Journey to the West, but it seems far from the style of A Dream of Red Mansions. But the creator of the absurd Bao Yu has made a Longjiang play with a dream of Red Mansions. This perspective is a great innovation. In terms of specific artistic expression, the creators merged the humanistic spirit of the Grand View Garden with the cultural sentiment of Guandong, and thus convincingly molded the characters of the Grand View Garden, such as Jia Baoyu of Guandong. For example, the Peach Blossom Dance in the prologue, the stone-burning dance in the fifth scene, the snowflake dance in the ending, and the performances with strong local characteristics in the play are all successful examples of the ingenious integration of Daguanyuan's humanistic sentiment and Guandong's cultural sentiment.

\section{Conclusion}

To sum up, a play (content); a feature (form); an innovation; a talent; an opening up, is the five elements of the construction and development of Longjiang Opera. These five elements are perfect, and audience problems, market problems and brand problems will be solved. In more than 40 years of historical development, generation after generation, represented by Bai Shuxian, Longjiang opera artists with hard work and wisdom sweat, finally cultivated Longjiang opera, a spring blossom on the land of Guandong, and handed in a satisfactory answer to their parents and elders. Nowadays, they are still thinking deeply on the way forward: how to create and perfect the style of singing music, how to create and perfect the characteristics of acting performance, how to grasp the combination of tradition and modern, inheritance and innovation, how to explore modern themes, reflect the spirit of the times... Longjiang opera is in the ascendant and has a long way to go. We wish the flowers of Longjiang opera on the fertile black land, and the spring breeze in the new era 
will be more varied.

\section{Acknowledgments}

Fund Project: Heilongjiang Philosophy and Social Science Planning Project:

Project Number: 18YSE621;

Project Name: Longjiang Opera and Art College Co-construction and Mutual Support Development Prospect Research

\section{References}

[1] Li Na. On the inheritance and development of Longjiang Opera under the new normal, [J]. art review, 2017 (06): 130-134.

[2] QiuYupeng. On the large-scale Longjiang drama "Mulan legend" [D]. Harbin Normal University, 2017.

[3] Li Na. On the artistic features of different stages of Longjiang drama [J]. art education, 2015 (12): $180-181$.

[4] Liu di. Inheritance and development of the two person circle in Longjiang drama [D]. Central Conservatory of Music, 2010.

[5] Li Mingming. Rational thinking on the construction and development of Longjiang opera [J]. art 100, 2003 (03): 5$8+20$. 